\title{
Cuando los delitos son episodios sociales
}

\author{
A. M. MARTÍN RODRÍGUEZ \\ Universidad de La Laguna

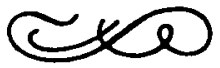

\section{Resumen}

En este trabajo se estudia la percepción del delito desde la perspectiva de los episodios sociales. Los objetivos más importantes de nuestra investigación fueron: a) aislar y comparar las dimensiones que subyacen a la percepción del delito en tres grupos de sujetos: delincuentes institucionalizados, profesionales del sistema penal y grupo de personas no vinculadas al sistema penal; $y \quad b)$ ver si existen diferencias en la percepción de tres de los tipos de delitos establecidos en el Código Penal español. La muestra e'stuvo compuesta por un total de 272 sujetos, todos ellos varones, procedentes de los tres grupos en estudio. Las puntuaciones dadas por estos sujetos a 22 episodios delictivos fueron sometidas a escalamiento multidimensional. Se obtuvo un espacio multidimensional de cuatro dimensiones para cada uno de los grupos y se constataron diferencias entre ellos. Las puntuaciones de los episodios en cada dimension permitieron discriminar de forma significativa entre los tres tipos de delitos. Los resultados se discuten integrando la perspectiva de los cpisodios sociales y la teoria de la atribución.

Palabras clave: Delitos, episodio social.

\section{Crimes as social episodes}

\section{Abstract}

The perception of the offense was studied from the point of view of social cpisodes, in order to: a) represent and compare the dimensions underlying the perception of the offense in three group of subjects: institutionalized delinquents, criminal system professionals and a group of pcople unrelated to the criminal system; and $b$ ) see whether there are any differences in the perception of three categories of offenses established in the Spanish Criminal Code. The sample was composed of 272 subjects, all of them males, proceeding from the three groups studied. The judgments given by these subjects on 22 offense episodes were analyzed by multidimensional scaling. A four dimensional space was found for each of the groups, and differences between groups were detected. The scores of the episodes in each dimension allowed for significant discrimination among the three categories of offenses. The results are discussed integrating the perspective of the social episodes and the theory of attribution.

Keywords: Offenses, social episodes.

Agracecimientos: Esta investigación fue llevada a cabo gracias a una beca de Formación del Personal Investigador del MEC. El manuscrito se completó mientras se disfrutaba de una beca de Reincorporación a España de Doctores y Tecnólogos, también del MEC.

Agradezco la inestimable ayuda que mis compañeros del área de Psicología Social de la Universidad de La Laguna me prestaron en la revisión del manuscrito.

Dirección del autor: Departamento de Psicología Cognitiva, Social y Organizacional. Universidad de La Laguna. Tenerife. 
Todo el mundo sabe ya que la delincuencia es uno de los problemas sociales más importantes de la época actual. Se ha repetido hasta la saciedad que «el poder destructivo del hombre para el hombre» ha ido incrementándose durante los últimos tiempos, y que la violencia se ha introducido incluso en el hogar de los "menos sospechosos». Lo que no se ha dicho es cómo vemos esa violencia nosotros, espectadores $y$, cada vez más, participantes de tales incidentes. Ni siquiera se ha intentado averiguar de qué forma se representan el delito los profesionales encargados de evaluarlo o aquellos que lo cometen. Evidentemente, si el fenómeno agresivo/delictivo ha cāmbiado, la percepción de las personas tiene que haberse visto afectada de algún modo; o viceversa.

Con relativa frecuencia se asume que la categorización de los incidentes agresivos constituye la base del sistema penal de un país. Muchos legisladores y algunos encargados de la administración de la justicia parecen estar convencidos de que la ley es un reflejo de las tradiciones y costumbres vigentes en la comunidad. Según estos defensores de la concepción naturalista del derecho, la ley es sólo la explicitación de la moral y valores que sustentan la sociedad y reproduce fielmente la percepción ciudadana del delito. Para ellos, el Orden Legal sería, en última instancia, la imagen tangible del Orden Social (ver Garzón y Seoane, 1988).

Existe otra concepción, más positivista, del derecho que considera la ley como el resultado de la actividad intencional de los gobernantes $\mathrm{y}$, por tanto, como un producto eminentemente político. Sin embargo, sigue habiendo juristas partidarios del primer enfoque $y$, lo que es peor, los estudios psicosociales sobre la percepción del delito parten exclusivamente de supuestos vinculados con esta concepción primitiva.

La Psicología Social ha dedicado mucha atención al tema de la delincuencia, pero se ha preocupado muy poco de la percepción del delito. Este hecho resulta sorprendente si tenemos en cuenta que la delincuencia se define en función de la ley vigente y ésta, como hemos dicho anteriormente, en general es presentada como un producto del consenso social. Las escasas investigaciones llevadas a cabo, la mayoría en Norteamérica, se limitan a comparar la gravedad de los delitos tal como es percibida por la opinión pública y la gravedad que se desprende de la ley. También se ha cuantificado la frecuencia de los delitos, la cantidad de castigo prescrito y se han elaborado estadísticas de todo tipo (ver Martín, 1987a).

Los trabajos sobre percepción de la conducta agresiva tampoco ayudan a esclarecer el tema de la percepción del delito, ya que se limitan a condensar las características de los actos agresivos en una simple dimensión de peligro social o gravedad, y a prescribir un continuo análogo para el castigo. Y eso a pesar de que se sabe, desde hace tiempo, que la gravedad percibida de un acto agresivo no es estática en el tiempo y que tampoco se relaciona consistentemente con la cantidad de castigo formalmente prescrito (ver Martín, 1984, 1987b).

En general, la mayoría de las investigaciones que analizan la percepción de la conducta delictiva y/o agresiva comparten cuatro errores de base (Forgas y cols., 1980, pág. 216):

1. Utilizan como estímulos un subgrupo arbitrario e ingenuamente pequeño de actos delictivos. 
2. Describen con simples etiquetas incidentes que la mayoría de los sujetos no han vivido personalmente o utilizan términos legales con los que éstos no están familiarizados.

3. Los investigadores, a excepción de Sherman y Dowle (1974), y Forgas (1980; Forgas y cols., 1980), preseleccionan una dimensión de juicio: la gravedad.

4. Los incidentes estudiados pertenecen tanto a categorías agresivas como no agresivas.

Para subsanar estos problemas habría que partir de una concepción diferente de los incidentes agresivos/delictivos y utilizar una metodología acorde con el nuevo enfoque. Desde nuestro punto de vista, esta concepción consistiría en considerar que tanto los actos agresivos como los delictivos son interacciones sociales. Tradicionalmente los psicólogos sociales han preferido centrarse en las características que diferencian los incidentes delictivos/agresivos de otras formas de comportamiento social, y no en los aspectos comunes. Las conductas agresivas y delictivas se conciben como formas del comportamiento antisocial por excelencia y, como tales, se sitúan en el polo opuesto de «lo social». Sin embargo, las distintas formas de agresión, e incluso los delitos, encajan perfectamente en cualquiera de las definiciones de conducta social al uso.

Por lo general, y salvo raras excepciones, esta tendencia segregacionista ha llevado a olvidar también que las conductas agresivas y delictivas se producen en un contexto y en un tiempo determinado, formando parte de la vida cotidiana. Pero la capacidad del ser humano para dar sentido al mundo que le rodea alcanza también a tales interacciones y las hace susceptibles de categorización social. De unos años a esta parte, determinados autores vienen utilizando el término episodios sociales para referirse a las categorías de interacciones sociales, independientemente del tipo de tales interacciones.

Las categorías de interacciones agresivas/delictivas pueden considerarse, en principio, dos tipos más de episodios sociales y, como tales, definirse como «representaciones cognitivas de secuencias de interacción estereotípicas, propias de un ambiente cultural dado» (Forgas, 1979a, pág. 15). Surgen, por lo tanto, de la actividad cognitiva de los miembros de una cultura y no de los individuos aislados. La integración de los procesos sociales, por un lado, y cognitivos, por el otro, que están en su origen se produce en virtud de las interacciones cotidianas (Forgas, 1982). Formulaciones muy semejantes a ésta pueden encontrarse en los trabajos de los teóricos del Interaccionismo Simbólico (p.e. Mead, 1934; Blumer, 1969) y de aquellos que se preocupan del origen de algunas categorias naturales (Rosch y cols., 1976).

La dualidad individual-social, junto con la naturaleza «implícita» de los episodios agresivos/delictivos hace que su estudio - así como el de cualesquiera otros episodios sociales - requiera de un procedimiento de investigación y de una técnica de análisis estadístico muy particulares. El procedimiento, toma como partida el método de los incidentes críticos de Flanagan (1954) y consiste, sucintamente, en obtener una muestra representativa del universo real de interacciones sociales objeto de investigación. La técnica de análisis estadístico es el escalamiento multidimensional, especial- 
mente el «Individual Differences Multidimensional Scaling» (INDSCAL) de Carroll y Chang (1970) (ver Camacho y Hernández, 1985; Forgas, 1979b; Kruskal y Wish, 1978; Schiffman y cols., 1981).

Desde la perspectiva de los episodios sociales sólo se han llevado a cabo dos estudios directamente relacionados con esta investigación: uno en el que se analiza la percepción del delito (Forgas, 1980), y otro sobre la percepción de la conducta agresiva (Forgas y cols., 1980). Sin embargo, en relación a otros tipos de episodios sociales existe multitud de estudios empíricos (p.e. Forgas 1976, 1978, Forgas y Bond, 1985), revisiones y discusiones teóricas sobre la naturaleza, origen y características de los mismos (p.e. Forgas, 1979a, 1981a, 1982), que nos han servido como marco de referencia más general, y a los que remitimos para un tratamiento más exhaustivo y detallado del tema.

Tomadas conjuntamente, las investigaciones sobre episodios delictivos/agresivos a la que hacíamos referencia en el párrafo anterior (Forgas, 1980; Forgas y cols., 1980), sugieren que:

1. La percepción de episodios delictivos y agresivos es multidimensional.

2. Las dimensiones que subyacen a la percepción de los episodios delictivos son diferentes de las que subyacen a la percepción de episodios agresivos.

3. Se pueden establecer distintos tipos de episodios a partir de las dimensiones del espacio perceptivo.

4. Algunas características individuales de los sujetos se relacionan con su percepción de los episodios.

Habría, no obstante, que contemplar estas conclusiones con cierta cautela, puesto que:

1. Los estímulos utilizados por Forgas (1980) son simples etiquetas y no episodios sociales en el sentido estricto del término.

2. Ambos estudios han sido llevados a cabo en el mundo anglosajón, en el cual no sólo la cultura es diferente sino también el sistema legal ("Common Law").

3. Las personas a partir de las cuales se obtienen los datos son estudiantes universitarios.

4. No se tienen en cuenta los estudios sobre percepción de la conducta agresiva realizados desde el área del juicio moral y la teoría de la atribución.

Nuestra investigación pretende subsanar algunos de estos problemas y contestar a dos cuestiones que nos parecen fundamentales a la hora de abordar la percepción del delito.

La primera se refiere a las dimensiones que subyacen a la percepción que personas vinculadas y no vinculadas al sistema penal tienen de los episodios delictivos. Nos interesa saber qué variables intervienen a la hora de evaluar una transgresión, cómo se agrupan y si cada uno de los grupos seleccionados tiene entidad social suficiente para producir una estructura perceptiva propia.

En segundo lugar, queremos saber cómo se relaciona la ley, tal y como aparece recogida en el Código Penal, con la percepción de los episodios de- 
lictivos. Concretamente nos centraremos en tres tipos de delitos, con el objeto de ver si las personas que toman parte en la investigación reproducen la clasificación establecida por el legislador y, de ser así, en base a qué criterios llevan a cabo la diferenciación.

Estudiaremos episodios delictivos exclusivamente -aunque hagamos referencia a aquellos estudios sobre percepción de la conducta agresiva que sean relevantes en la discusión de nuestros resultados- por dos razones. En primer lugar, los estudios de Forgas sugieren que el espacio perceptivo de ambos tipos de conducta es diferente. En segundo lugar, la conducta agresiva y la conducta delictiva son, por definición, distintas.

En síntesis, los objetivos de nuestra investigación podrían enumerarse de la siguiente forma:

1. Estudiar las dimensiones que subyacen a la percepción del delito en tres grupos de sujetos: delincuentes, profesionales del sistema penal y grupo de personas no vinculadas al sistema penal.

2. Ver si existen diferencias en la percepción de tres de los tipos de delitos establecidos en el Código Penal español: delitos contra la propiedad, contra las personas y contra la honestidad.

\section{METODO}

\section{Sujetos}

En este estudio tomaron parte 272 sujetos, todos ellos varones, procedentes de 3 grupos sociales diferentes: delincuentes institucionalizados, profesionales del sistema penal y un grupo de personas no vinculadas al sistema penal.

La muestra de delincuentes estuvo compuesta por 90 varones seleccionados al azar del total de 404 internos que cumplian condena o estaban en espera de sentencia en el Centro de Detención y Cumplimiento de S. C. de Tenerife. Estos internos procedían de 7 de los 9 grupos existentes en la prisión, exceptuando Enfermería y Mujeres. La media de edad de esta muestra fue de 26,2 y la desviación típica 8,2.

Los profesionales que participaron en la investigación fueron 90 en total: 30 policías, 30 funcionarios de prisiones y 30 jueces. Los policías pertenecian todos a la policía judicial. Los funcionarios de prisiones fueron seleccionados al azar entre todos los que prestaban servicio en el interior de la institución, en contacto directo con los internos. Los jueces procedían de las islas de Tenerife, La Palma, La Gomera y El Hierro. La media de edad de esta muestra fue 36,2 y la desviación típica 11,8 .

También obtuvimos datos de 92 sujetos no vinculados al sistema penal. Este grupo estuvo compuesto por personas que asistían a los cursos de formación del INEM, en diversas localidades de la isla de Tenerife. Poseían un nivel sociocultural semejante al de los delincuentes institucionalizados pero, a diferencia de éstos, se encontraban en libertad. La mitad de ellos trabajaba y la otra mitad estaba en paro. Preferimos esta muestra, frente a una de estudiantes universitarios, porque consideramos que constituía un grupo de comparación más válido respecto a los delincuentes. La media de edad del grupo fue 24,85 y la desviación típica 7,67. 


\section{Instrumento}

De acuerdo con la orientación teórica de este trabajo es fundamental obtener una amplia gama de episodios delictivos experimentados personalmente o, por lo menos, familiares para los sujetos. Por esta razón, se desarrollaron una serie de sesiones de grupo con 70 internos (en grupos de 10) de la Prisión Provincial de Las Palmas de Gran Canaria (Salto de El Negro) y 30 estudiantes de FP2. En ellas se obtuvieron descripciones de episodios delictivos, bien experimentados personalmente por los sujetos, ya sea como observadores, como agresores o como víctimas, o bien conocidos a través de alguien próximo al hecho. En todo caso, se trataba de eventos típicos del ambiente en el que habitualmente se desenvolvía el narrador. También se les pidió que enumeraran qué aspectos tendrían en cuenta para juzgar episodios como los narrados.

La revisión de todo este material permitió seleccionar los 22 episodios delictivos que aparecen en la tabla I, como los más frecuentes y típicos.

Se seleccionaron además 20 escalas bipolares, que aparecen en la tabla II, también a partir de las declaraciones de los sujetos. Los criterios de selección fueron la frecuencia y la relevancia para los sujetos y los objetos juzgados, a través de las situaciones.

Muchas de estas escalas son similares a las utilizadas en la literatura previa sobre juicio moral, atribución de responsabilidad y percepción de episodios, y han demostrado ser útiles a la hora de diferenciar entre distintos tipos de conductas agresivas.

Se prefirió el uso de las escalas bipolares frente a otros métodos de recogida de datos para el escalamiento multidimensional por dos motivos. En primer lugar, los métodos alternativos requerían más tiempo para su administración (por ejemplo, comparación por pares) o una aplicación individual (por ejemplo, ordenación de tarjetas). Además, se supone que con un número bastante grande de escalas los sujetos no se verán influidos por ellas en su percepción de episodios. Asimismo, estas escalas pueden utilizarse posteriormente como indicadores en la interpretación de las dimensiones obtenidas en el INDSCAL.

\section{Procedimiento}

Para la recogida de los datos se siguieron los pasos clásicos de la administración de cualquier prueba de papel y lápiz, teniendo muy en cuenta las características de las tres muestras de sujetos. Los delincuentes y el grupo no vinculado al sistema penal cumplimientaron las escalas en pequeños grupos bajo una estrecha supervisión. Los profesionales contestaron a los ítems individualmente. En todos los casos se comprobó que las instrucciones habian sido entendidas y respetadas al máximo.

Se pidió a los sujetos que puntuaran cada uno de los 22 episodios en todas y cada una de las 20 escalas, eligiendo para ello entre 7 intervalos. Estas puntuaciones se transformaron en matrices de disimilaridades, que sirvieron de entrada al INDSCAL.

La transformación se llevó a cabo a través de la fórmula desarrollada por Cronbach y Gleserl (1952), Osgood y Suci (1952) y Wish y cols. (1976). 


\section{1}

TABI.A I

Lista de los 22 episodios delictivos utilizados como estimulos en este estudio

1. "Un grupo de extranjeros trafica con droga utilizando un yate. Cuando los detuvieron encontraron 5.000 kilos de chocolate en el yate."

2. "Dos moros le roban a una chica $c$ intentan violarla. Entonces interviene otra persona que los golpea con una barra de hierro y les roba a ellos."

3. "Una banda de extranjeros rapta chicas y las vende para la trata de blancas."

4. "Una chica fue a una fiesta en su Instituto. Al día siguiente, acusó de violación a un profesor que la había llevado a su casa porque se sentía mal. La chica afirmó que le habian dado bebidas y grifa para que fumara. Algunas personas dijeron que habian visto cómo el profesor volvía a la fiesta."

5. "Un hombre llegó borracho a su casa y tuvo una pelea con su padre y su tío. Como se vio en inferioridad de condiciones cogió una escopeta y, cuando su padre intentó quitársela, el arma se disparó y alcanzó a su madre que escuchaba detrás de la puerta. La mujer murió del turo."

6. «Un padre violó repetidamente a sus tres hijas durante varios años, con la excusa de darles educación sexual."

7. “Dos hermanos querían la herencia de su padre. Uno de ellos tenía un hijo bobo. Le dieron una escopeta, lo llevaron a un huerto donde estaba el vicjo y le dijeron que lo matara. Después, los dos hermanos se quedaron con la herencia y el bobo fue a la cárcel."

8. "Un chico le robó a un coreano. A los dos meses el coreano volvió, reconoció al chico y le dio siete puñaladas. No lo mató, pero estuvo muy mal.»

9. "Más de treinta coreanos le pegan a otro de ellos. Le dan patadas en la cabeza y lo golpean contra el piso hasta que lo matan."

10. "Un grupo de jóvenes, después de beber y fumar grifa, arman jaleo en un barco y entran en los camarotes de la gente."

11. «En una librería una persona se llevó una revista sin pagar. La escondió y luego disimuló.»

12. «Un hombre le dijo a otro que le vendía un tractor pequeño. Puso como condición que el dinero se lo diera por anticipado y, cuando lo tuvo, se marchó dejando al otro. $\sin$ tractor y sin dinero."

13. "Una scñora va por la calle con un bolso colgado del brazo. Un hombre aparece caminando, le da un tirón al bolso y se lo quita. La señora. se pone a gritar para que alguien lo detenga, pero el hombre desaparece entre la gente."

14. "Un chico iba conduciendo una moto por su derecha en una carretera. Al llegar a un cruce le sale al paso un camión que lo arrolla. El conductor del camión se da a la fuga dejándolo en el suelo y el chico muere del impacto sufrido."

15. "Varias personas le robaron a un hombre un talego y medio. Después lo secuestraron para quitarle cuatrocientos talegos más que tenia en el banco."

16. "Un hombre marroquí es acusado de malos tratos y de prostituir a su mujer."

17. "Un chico abrió un coche para robar el cassette y los altavoces. Después le quitó el freno de mano y lo dejó ir por la pendiente para que se chocara y hacerle la faena al dueño."

18. "Un chico cruza una montaña para ahorrarse camino. Dos grifientos, con pegamento, le sacan una navaja obligándole a darles todo lo que lleva, porque si no le rajan." (Con.)

19. "Unos chicos jóvenes desvalijan coches delante de un Instituto. Parecen estar organizados y actúan con gran soltura, a pleno día y delante de la gente."

20. "Un borracho le dio doce puñaladas a otra persona y la escondió entre unas tuneras.".

21. "Tres chicos, de 9 a 12 años. cogen las macetas que están en la entrada de un portón y las tiran al suelo rompiéndolas. Lo hicieron varias veces durante la madrugada y eran de la misma barriada."

22. "Un hombre se metió varias veces en unos almacenes para cargar de comida una furgoneta. Le llevaba parte a su familia y el resto lo vendía."

De acuerdo con dicha fórmula, la disimilitud $\sigma_{j k(s)}$ entre los episodios $j$ y $k$ en una escala s se define como:

$$
\sigma_{i k(m)}=\frac{1}{N} \vee \sum_{i=1}^{N}\left(X_{i j(m)}-X_{i k \cdot(m)}\right)^{2}
$$




\section{TABLA II}

Lista de las 20 escalas bipolares utilizadas en este estudio

1. Muy grave/nada grave: Gravedad del hecho.

2. Injustificable/justificable: Justificación del hecho.

3. En provecho propio/en provecho ajeno: Agresión personal/prosocial.

4. Sólo para hacer daño/para conseguir algo: Agresión hostil/instrumental.

5. Lo volverá a hacer/no lo volverá a hacer: Probabilidad conducta futura.

6. Mucho castigo/ningún castigo: Cantidad de castigo.

7. Consecuencias leves/consecuencias graves: Gravedad de las consecuencias.

8. Intencional/no intencional: Intencionalidad del hecho.

9. Provocado/no provocado: Provocación.

10. Hay relación entre el delito y la condena/no hay relación entre el delito y la condena: Relación hecho/castigo.

11. Con influencia del alcohol/sin influencia del alcohol: Alcohol/no alcohol.

12. Es probable que sea castigado/no es probable que sea castigado: Probabilidad del castigo.

13. Es raro que pase/es probable que pase: Probabilidad de ocurrencia.

14. Pasional/calculado: Emocional/no emocional.

15. El agresor es culpable/el agresor es inocente: Culpabilidad.

16. Me cae bien el agresor/no me cae bien: Simpatia hacia el agresor.

17. Sin violencia/con violencia: Violencia.

18. Empezó el agresor/empezó la víctima: Agresión iniciatoria/reactiva.

19. El agresor es responsable del daño/el agresor no es responsable del daño: Responsabilidad.

20. Me cae bien la víctima/no me cae bien la víctima: Simpatía hacia la víctima.

donde $\mathrm{X}_{\mathrm{ij}(\mathrm{s})}$ y $\mathrm{X}_{\mathrm{ik}(\mathrm{s})}$ son las puntuaciones dadas por cada sujeto i a los episodios $\mathrm{j} y \mathrm{k}$ en la escala $\mathrm{s}$, y $\mathrm{N}$ el número total de sujetos que puntuaron ese epidosio en esa escala.

Se llevaron a cabo una serie de INDSCAL en los que se emplearon como input 20 matrices correspondientes a las 20 escalas. En todos los casos se consideró la población como unidad de análisis.

Debido al procedimiento reiterativo tan complejo utilizado por el INDSCAL, algunas veces los resultados son soluciones no del todo óptimas y estables. Para asegurarnos de la estabilidad de nuestros resultados, se repitieron los análisis varias veces, modificando el número de dimensiones y las configuraciones aleatorias de entrada. El número de dimensiones elegido fue, a nuestro juicio, el más adecuado para representar, estadística y conceptualmente, la percepción de episodios delictivos en cada población. Procuramos, en todo caso, que este número fuera lo más pequeño posible y que explicara el mayor porcentaje de varianza posible, así como que nos proporcionara un índice de ajuste adecuado. A la hora de interpretar cada una de las dimensiones se tuvo en cuenta el peso de cada escala en esa dimensión del INDSCAL. Estos pesos son análogos a coeficientes de correlación parcial e indican la relación que existe entre una escala dada y las dimensiones del espacio perceptivo (Wish y cols., 1976).

A continuación presentamos los principales resultados obtenidos, especificando a qué grupo de sujetos corresponden. 


\section{RESULTADOS}

\section{El espacio perceptivo de los episodios delictivos}

\section{Delincuentes}

La solución de cuatro dimensiones nos pareció la óptima, en el caso de los delincuentes, según los criterios expuestos anteriormente. Esta solución explica un $52 \%$ de la varianza y tiene un índice de ajuste de 0,55 , lo cual, en este tipo de análisis, se considera aceptable. En la figura 1 se representa la distribución de los episodios delictivos en relación a las cuatro dimensiones del espacio perceptivo, mientras que la tabla III resume los principales resultados de este análisis.

TABLA III

Resumen de los principales resultados del INDSCAL en el grupo de los delincuentes (pesos de cada escala bipolar en, y varianza explicada por cada dimensión y por la solución total)

\begin{tabular}{|c|c|c|c|c|}
\hline Escalas & Gravedad & $\begin{array}{l}\text { Respon- } \\
\text { sabilidad }\end{array}$ & $\begin{array}{l}\text { Inten- } \\
\text { cionalidad }\end{array}$ & Atenuantes \\
\hline Gravedad del hecho... & 0,94 & 0,03 & 0,03 & 0,07 \\
\hline Justificación del hecho ......... & 0,34 & 0,20 & 0,06 & 0,33 \\
\hline Agresión personal/prosocial.................. & 0,09 & 0,59 & 0,14 & 0,30 \\
\hline Agresión hostil/Instrumental ................ & 0,08 & 0,04 & 0,05 & 0,86 \\
\hline Probabilidad conducta futura................ & 0,16 & 0,30 & 0,58 & 0,12 \\
\hline 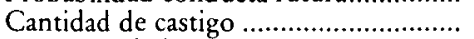 & 0,84 & 0,26 & 0,01 & 0,00 \\
\hline 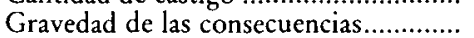 & 0,93 & 0,02 & 0,03 & 0,04 \\
\hline 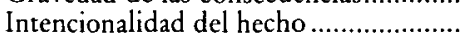 & 0,12 & 0,20 & 0,76 & 0,08 \\
\hline 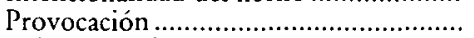 & 0,22 & 0,14 & 0,61 & 0,04 \\
\hline 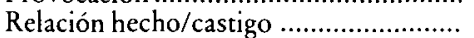 & 0,58 & 0,45 & 0,03 & 0,04 \\
\hline No alcohol/alcohol ............................... & 0,05 & $-0,01$ & 0,35 & 0,38 \\
\hline Probabilidad de castigo...................... & 0,64 & 0,15 & 0,05 & 0,01 \\
\hline 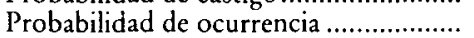 & 0,36 & 0,15 & 0,26 & 0,21 \\
\hline 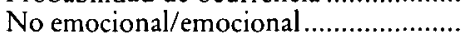 & 0,07 & 0,12 & 0,64 & 0,35 \\
\hline 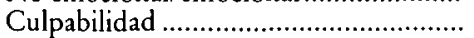 & 0,21 & 0,85 & 0,07 & 0,06 \\
\hline 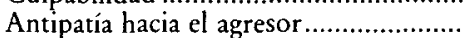 & 0,58 & 0,43 & 0,02 & 0,20 \\
\hline 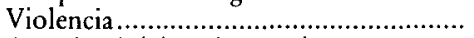 & 0,63 & 0,02 & 0,02 & 0,18 \\
\hline Agresión iniciatoria/reactiva ................. & 0,11 & 0,07 & 0,11 & 0,03 \\
\hline Responsabilidad .................................... & 0,13 & 0,78 & 0,16 & 0,09 \\
\hline Simpatía hacia la víctima...................... & 0,20 & 0,12 & 0,19 & 0,08 \\
\hline Varianza explicada ................................ & $22,35 \%$ & $12,24 \%$ & $10,21 \%$ & $7,09 \%$ \\
\hline
\end{tabular}

Varianza total explicada $=51,90 \%$. Indice de ajuste $=0,55$.

La primera dimensión, distingue entre episodios como robar comida (22), el hurto de una revista (11), romper macetas (21), que sugieren hechos poco graves, con consecuencias leves y que, según los delincuentes, merecen poco castigo y episodios como la trata de blancas (3), la violación por parte de un padre (6), asesinato por treinta coreanos (9), en el otro extremo de la gravedad del hecho, de las consecuencias y del castigo merecido.

Esta dimensión, que hemos llamado gravedad, explica un $22,3 \%$ y viene definida, fundamentalmente, por las escalas «muy grave/nada grave» $(0,94)$, «consecuencias graves/leves» $(0,93)$, "mucho castigo/poco castigo" $(0,85)$, «es improbable que sea castigado/es probable que sea castigado" $(0,64)$, "con violencia/sin violencia" $(0,63)$ y, en menor grado, por "no me cae bien el agresor/me cae bien" $(0,58)$, "hay relación entre el delito y la condena/no hay relación" $(0,58)$, «es probable que pase/es improbable que 


\section{4}

FIGURA 1
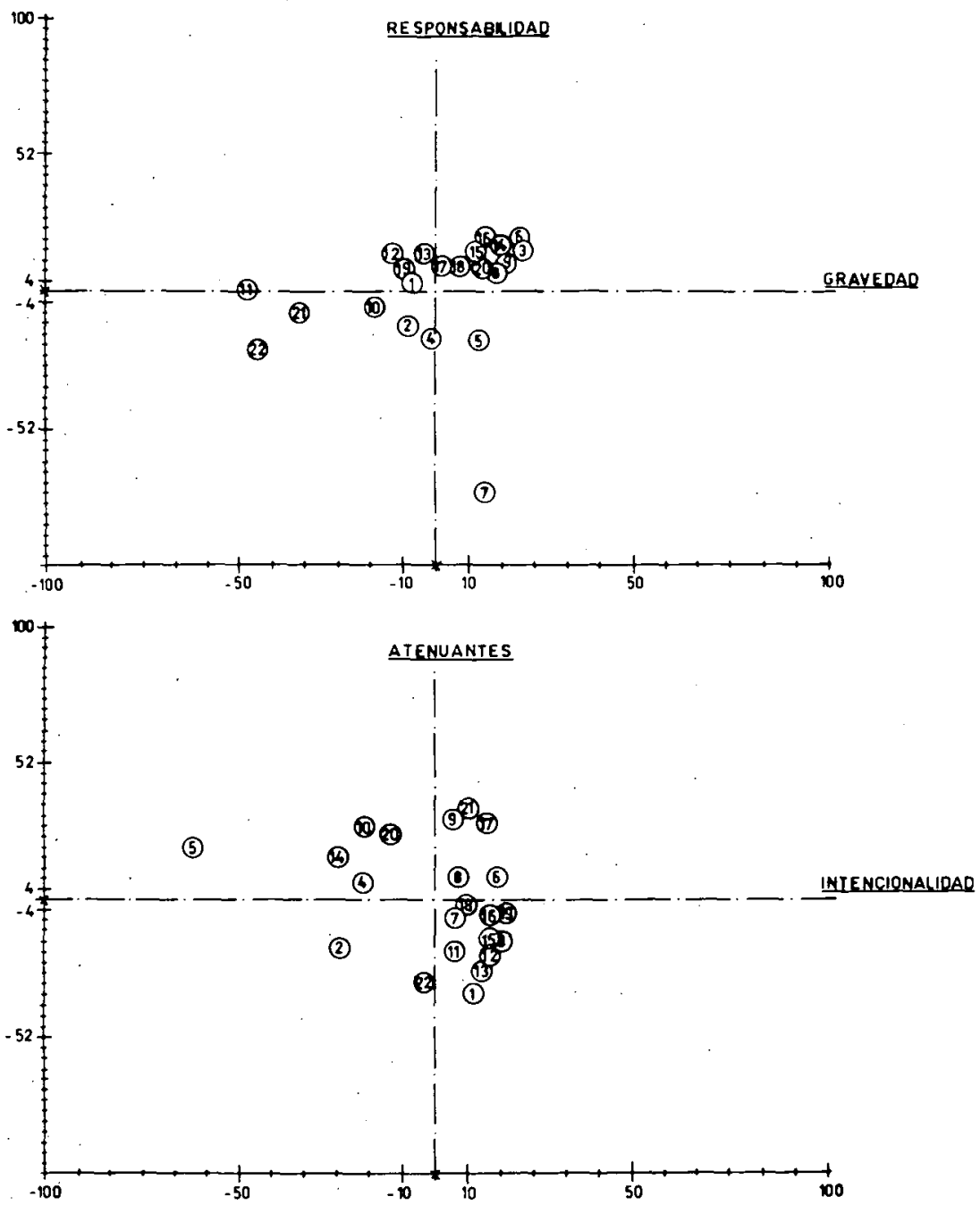

1. TRAFICO DE DROGAS

12. ESTAFA DEL TRACTOR

2. CHICO GOLPEA A MOROS

13. TIRON DE UN BOLSO

3. TRATA DE BLANCAS

6. PRE SUNTA VIOLACION

14. MUERTE DEL MOTORISTA

5. BORRACHO MATA A SU MADRE

15. SECUESTRO

16. MALOS TRATOS A LA MUJER

5. PADRE VIOLA A SUS HIJAS

17. ROBO Y DESTROZO DE UN COCHE

T.BOBO" MATA AL ABUELO

6. COREANO OUE SE VENGA

9. 30 COREANOS MATAN A UNO

18. NTMIDACION CON NAVAJA

19. ROBO DE COCHES

20. DORRACHO OUE APUŃ̃LLA

10. CHICOS ARMAN JALEO

11. HURTO DE REVISTA

21. CHICOS OUE ROMPEN MACETAS

22. ROBO EN UN ALMACEN

Representación gráfica de los 22 episodios delictivos en el espacio perceptivo de los delincuentes. 
pase» $(0,36)$, «injustificable/justificable» $(0,34)$ y «me cae bien la victima/me cae mal la victima».

La segunda dimensión permite distinguir entre episodios como el del retrasado mental que comete un crimen (7), y el del marido que prostituye y maltrata a la esposa (16). Esta dimensión, responsabilidad, explica un $12,2 \%$ de la varianza y viene definida por las escalas "culpable/inocente" $(0,85)$, «agresor responsable/no responsable» $(0,78)$ y «en provecho propio/en provecho ajeno» $(0,59)$.

La tercera dimensión muestra en uno de sus extremos el episodio del hombre que mata a su madre sin querer (5) y, en el otro, el episodio en el que se describe un caso de trata de blancas (3), o el de la estafa (12). Esta dimensión, definida como intencionalidad, explica un $10,2 \%$ de la varian$\mathrm{za}$ y es reflejo de las escalas "intencional/no intencional" $(0,77)$, "calculado/pasional" $(0,64)$, "no provocado/provocado" $(0,61)$ y, en menor grado, "lo volverá a hacer/no lo volverá a hacer» $(0,58)$ y "empezó el agresor/empezó la víctima» $(0,12)$.

La cuarta dimensión sitúa en los extremos el episodio de tráfico de drogas a gran escala (1) o el de robo de comida (22), frente al episodio en que los coreanos asesinan a uno de ellos (9), o el episodio en que se destrozan macetas (21). Esta dimensión, atenuantes, explica un 7,1\% de la varianza y está definida por las escalas "sólo para hacer daño/para çonseguir algo" $(0,86)$ y «sin influencia del alcohol/con influencia del alcohol» $(0,38)$. Aquí la escala "hostil/instrumental" define la dimensión claramente. Los primeros episodios se perciben como instrumentales y los últimos como hostiles.

Se constataron ligeras correlaciones entre algunas de las dimensiones: gravedad y responsabilidad $(0,21)$, gravedad y atenuantes $(0,30)$, intencionalidad y responsabilidad $(0,33)$ y atenuantes e intencionalidad $(-0,32)$.

\section{Profesionales}

En los profesionales, la solución de cuatro dimensiones explica un $58 \%$ de la varianza y posee un índice de ajuste de 0,60. En la figura 2 se representa la distribución de los episodios delictivos en relación a las cuatro dimensiones del espacio perceptivo, mientras que la tabla IV resume los principales resultados de este análisis.

La primera dimensión distingue entre los episodios como el del hurto de una revista (11) o el de la rotura de macetas (21), de la riña tumultuaria con resultado de muerte (9), la violación repetida de un padre a sus tres hijas (6) o la trata de blancas (3). Esta dimensión recibe el nombre de gravedad, explica un $20,5 \%$ de la varianza y está definida por las escalas «muy grave/nada grave» $(0,97)$, «consecuencias graves/consecuencias leves» $(0,96)$, "mucho castigo/ningún castigo» $(0,92)$, «es improbable que sea castigado/es probable que sea castigado" $(0,72) \mathrm{y}$, en menor medida, por «hay relación entre el delito y la condena/no hay relación" $(0,51)$, «injustificable/justificable» $(0,43)$ y "con violencia/sin violencia» $(0,39)$.

La segunda dimensión permite distinguir entre el homicidio involuntario de la madre (5), el homicidio bajo los efectos del alcohol (20) o la imprudencia punible del accidente en que murió el motorista (14), y el tráfico de drogas (1), la trata de blancas (3) o el tirón (13). Esta dimensión explica un $16,4 \%$ de la varianza y se denomina intencionalidad. Las escalas a partir de las cuales hemos elegido su nombre son: "calculado/pasional" $(0,86)$, 
FIGURA 2
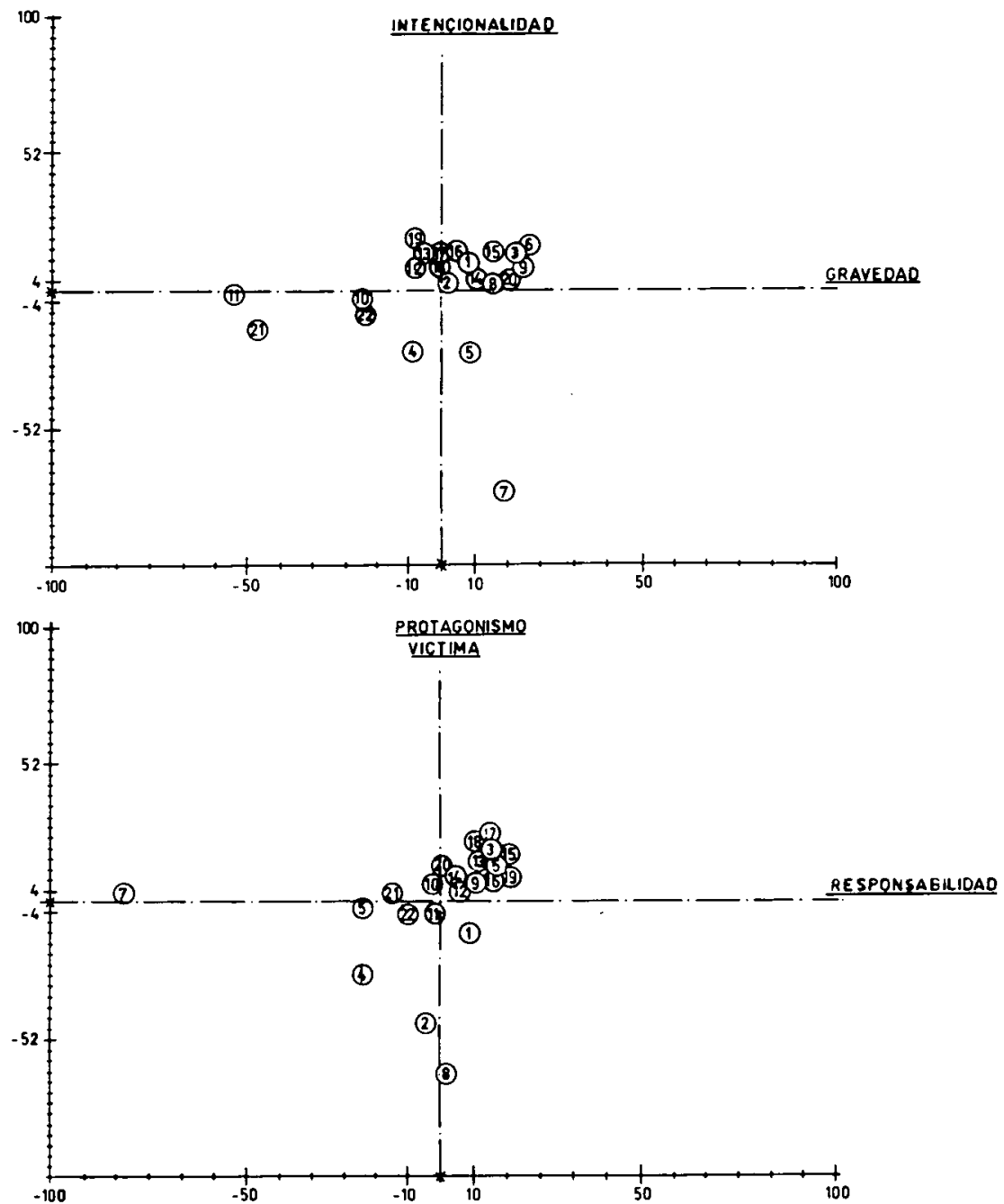

1. TRAFICO DE DROGAS

12. ESTAFA DEL TRACTOR

2. CHICO GOLPEA A MOROS

13. TIRON DE UN BOLSO

3. TRATA DE BLANCAS

14. MUERTE DEL MOTORISTA

6. PRESUNTA VIOLACION

15. SECUESTRO

5. BORRACHO MATA A SU MADRE

16. MALOS TRATOS A LA MUJER

6. PADRE VIOLA A SUS HIJAS

7.BOBO" MATA AL ABUELO

17. ROBO Y DESIROZO DE UN COCHE

8. COREANO OUE SE VENGA

18. INTIMIDACION CON NAVAJA

9. 30 COREANOS MATAN A UNO

19. ROBO DE COCHES

20. BORRACHO DUE APUÑALA

10. CHICOS ARMAN JALEO

21. CHICOS OUE ROMPEN MACETAS

11. HURTO OE REVISTA

22. ROBO EN UN ALMACEN

Representación gráfica de los 22 episodios delictivos en el espacio perceptivo de los profesionales del sistema penal. 
TABLA IV

Resumen de los principales resultados del INDSCAL en el grupo de los profesionales (pesos de cada escala bipolar en, y varianza explicada por cada dimensión y por la solución total)

\begin{tabular}{|c|c|c|c|c|}
\hline Escalas & Gravedad & $\begin{array}{l}\text { Inten- } \\
\text { cionalidad }\end{array}$ & $\begin{array}{l}\text { Respon- } \\
\text { sabilidad }\end{array}$ & $\begin{array}{c}\text { Protagonis- } \\
\text { mo } \\
\text { víctima }\end{array}$ \\
\hline Gravedad del hecho. & 0,97 & 0,02 & 0,03 & 0,03 \\
\hline Justificación del hecho. & 0,43 & 0,14 & 0,40 & 0,21 \\
\hline Agresión personal/prosocial.. & 0,05 & 0,52 & 0,53 & 0,03 \\
\hline Agresión hostil/Instrumental ............... & 0,02 & 0,33 & $-0,00$ & 0,06 \\
\hline Probabilidad conducta futura ..................... & 0,07 & 0,68 & 0,39 & 0,08 \\
\hline 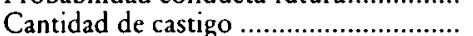 & 0,92 . & $-0,00$ & 0,18 & 0,03 \\
\hline Gravedad de las consecuencias.................. & 0,96 & 0,03 & 0,00 & 0,01 \\
\hline Intencionalidad del hecho & 0,04 & 0,79 & 0,08 & $-0,00$ \\
\hline 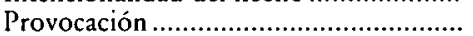 & 0,15 & 0,51 & 0,16 & 0,31 \\
\hline Relación hecho/castigo .......................... & 0,50 & 0,23 & 0,26 & 0,07 \\
\hline No alcohol/alcohol ................................... & 0,02 & 0,68 & $-0,04$ & 0,00 \\
\hline Probabilidad de castigo .......................... & 0,72 & 0,10 & 0,16 & 0,03 \\
\hline Probabilidad de ocurrencia..... & 0,30 & 0,46 & 0,22 & 0,06 \\
\hline No emocional/emocional .......................... & 0,04 & 0,86 & 0,00 & 0,04 \\
\hline 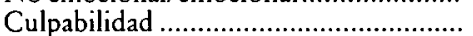 & 0,15 & 0,06 & 0,88 & 0,02 \\
\hline Antipatia hacia el agresor.......................... & 0,24 & 0,00 & 0,67 & 0,29 \\
\hline 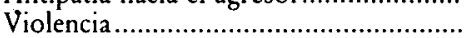 & 0,39 & 0,09 & $-0,00$ & 0,00 \\
\hline Agresión iniciatoria/reactiva ................. & 0,05 & 0,11 & 0,09 & 0,82 \\
\hline 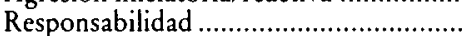 & 0,13 & 0,03 & 0,88 & 0,02 \\
\hline 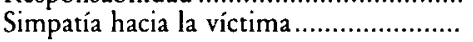 & 0,08 & 0,06 & 0,02 & 0,74 \\
\hline 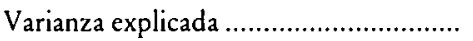 & $20,59 \%$ & $16,45 \%$ & $14,32 \%$ & $7,49 \%$ \\
\hline
\end{tabular}

Varianza total explicada $=58,87 \%$. Indice de ajuste $=0,60$.

«intencional/no intencional» $(0,79)$, «sin influencia del alcohol/con influencia» $(0,68)$, «lo volverá a hacer/no lo volverá a hacer» $(0,68)$ y, en menor medida, por «no provocado/provocado» $(0,51)$, «es probable que pase/es raro que pase» $(0,46)$ y «sólo para hacer daño/para conseguir algo» $(0,33)$.

En el caso de la tercera dimensión tendríamos, por un lado, episodios como el del padre que viola a sus hijas (6), el secuestro (5) o la trata de blancas (3), frente al asesinato cometido por un retrasado mental (7) o el homicidio involuntario de la madre (5). Denominamos responsabilidad a esta dimensión, que explica un $14,3 \%$ de la varianza, ya que las escalas que más puntúan en ella son: «el agresor es responsable/el agresor no es responsable» $(0,88)$, "el agresor es culpable/inocente» $(0,88)$, «me cae mal el agresor/me cae bien" $(0,67)$ y «en provecho propio/en provecho ajeno" $(0,53)$.

La cuarta dimensión separa episodios como el del coreano que apuñala a quien le había robado (8) o el del chico que golpea y roba a quienes robaban a otra persona (2), y episodios como el de la trata de blancas (3), la violación por parte del padre (6), el secuestro (15) o el robo con intimidación (18). Esta dimensión está integrada por escalas «empezó el agresor/empezó la víctima" $(0,83)$ y "me cae bien la víctima/me cae mal» $(0,74)$, explica un $7,49 \%$ de la varianza y se ha denominado protagonismo de la victima.

Las correlaciones entre dimensiones fueron pequeñas, pero nos parece interesante resaltar las dos siguientes: responsabilidad $e$ intencionalidad $(0,32)$, y responsabilidad y protagonismo de la víctima $(0,25)$. 
Grupo no vinculado al sistema penal

En el grupo de personas no relacionadas con el sistema penal, el porcentaje de la varianza explicado por la solución de cuatro dimensiones es del $55,4 \%$, mientras el índice de ajuste se sitúa en $0,57 \%$. En la figura 3 se representa la distribución de los episodios delictivos en relación a las cuatro dimensiones del espacio perceptivo, mientras que la tabla $\mathrm{V}$ resume los principales resultados de este análisis.

Si examinamos el gráfico anterior podemos ver cómo la primera dimensión distingue entre episodios como el hurto de una revista (11) y la violación repetida de un padre a sus hijas (6), o la riña tumultuaria con resultado de muerte (9). El porcentaje de varianza explicado en este caso es de un $20 \%$ de la varianza, mientras que las escalas que definen la dimensión son: "consecuencias graves/consecuencias leves» $(0,93)$, "muy grave/nada grave» $(0,93)$, «mucho castigo/poco castigo" $(0,79)$, «es improbable que sea castigado/es probable» $(0,73)$, «con violencia/sin violencia» $(0,62)$, «hay relación entre el delito y su condena/no hay relación» $(0,57)$ e «injustificable/justificable» $(0,39)$. Por ello hemos elegido el nombre de gravedad para designar a esta dimensión.

La segunda dimensión distingue entre episodios como el homicidio por parte de un retrasado mental (7), o por un borracho que disparó sin querer matar a nadie (5) y el padre que viola a sus hijas repetidamente (6), el chico

\section{TABLA V}

Resumen de los principales resultados del INDSCAL en el grupo de personas no vinculadas al sistema penal (pesos de cada escala bipolar en, y varianza explicada por cada dimensión y por la solución total)

\begin{tabular}{|c|c|c|c|c|}
\hline Escalas & Gravedad & $\begin{array}{l}\text { Respon- } \\
\text { sabilidad }\end{array}$ & $\begin{array}{l}\text { Inten- } \\
\text { cionalidad }\end{array}$ & $\begin{array}{c}\text { Protagonis- } \\
\text { mo } \\
\text { victima }\end{array}$ \\
\hline Gravedad del hecho. & 0,93 & 0,05 & 0,06 & 0,09 \\
\hline Justificación del hecho ... & 0,39 & 0,28 & 0,14 & 0,21 \\
\hline Agresión personal/prosocial.. & 0,09 & 0,67 & 0,23 & 0,01 \\
\hline Agresión hostil/Instrumental ................ & 0,07 & 0,04 & 0,21 & 0,10 \\
\hline Probabilidad conducta futura .................. & 0,09 & 0,24 & 0,72 & 0,13 \\
\hline Cantidad de castigo .................... & 0,79 & 0,38 & 0,02 & 0,08 \\
\hline Gravedad de las consecuencias. & 0,93 & 0,01 & 0,07 & 0,05 \\
\hline Intencionalidad del hecho ..................... & 0,00 & 0,25 & 0,79 & 0,00 \\
\hline 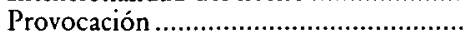 & 0,12 & 0,08 & 0,62 & 0,23 \\
\hline Relación hecho/castigo ......................... & 0,57 & 0,45 & 0,05 & 0,13 \\
\hline No alcohol/alcohol ................................... & 0,07 & $-0,00$ & 0,47 & 0,07 \\
\hline Probabilidad de castigo ............................. & 0,73 & 0,13 & 0,08 & 0,12 \\
\hline Probabilidad de ocurrencia ......................... & 0,19 & 0,23 & 0,43 & 0,26 \\
\hline No emocional/emocional ............................ & 0,09 & 0,04 & 0,70 & 0,17 \\
\hline 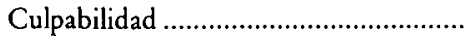 & 0,17 & 0,87 & 0,07 & 0,11 \\
\hline Antipatía hacia el agresor.......................... & 0,32 & 0,53 & 0,04 & 0,25 \\
\hline 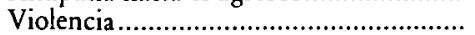 & 0,61 & $-0,01$ & 0,13 & 0,07 \\
\hline Agresión iniciatoria/reactiva ................. & 0,04 & 0,05 & 0,07 & 0,87 \\
\hline 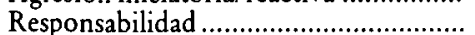 & 0,07 & 0,87 & 0,09 & 0,10 \\
\hline Simpatia hacia la víctima .......................... & 0,16 & $-0,01$ & 0,01 & 0,55 \\
\hline 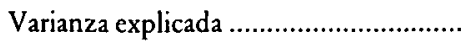 & $20,25 \%$ & $14,67 \%$ & $13,17 \%$ & $7,29 \%$ \\
\hline
\end{tabular}

Varianza total explicada $=55,40 \%$. Indice de ajuste $=0,57$. 
FIGURA 3
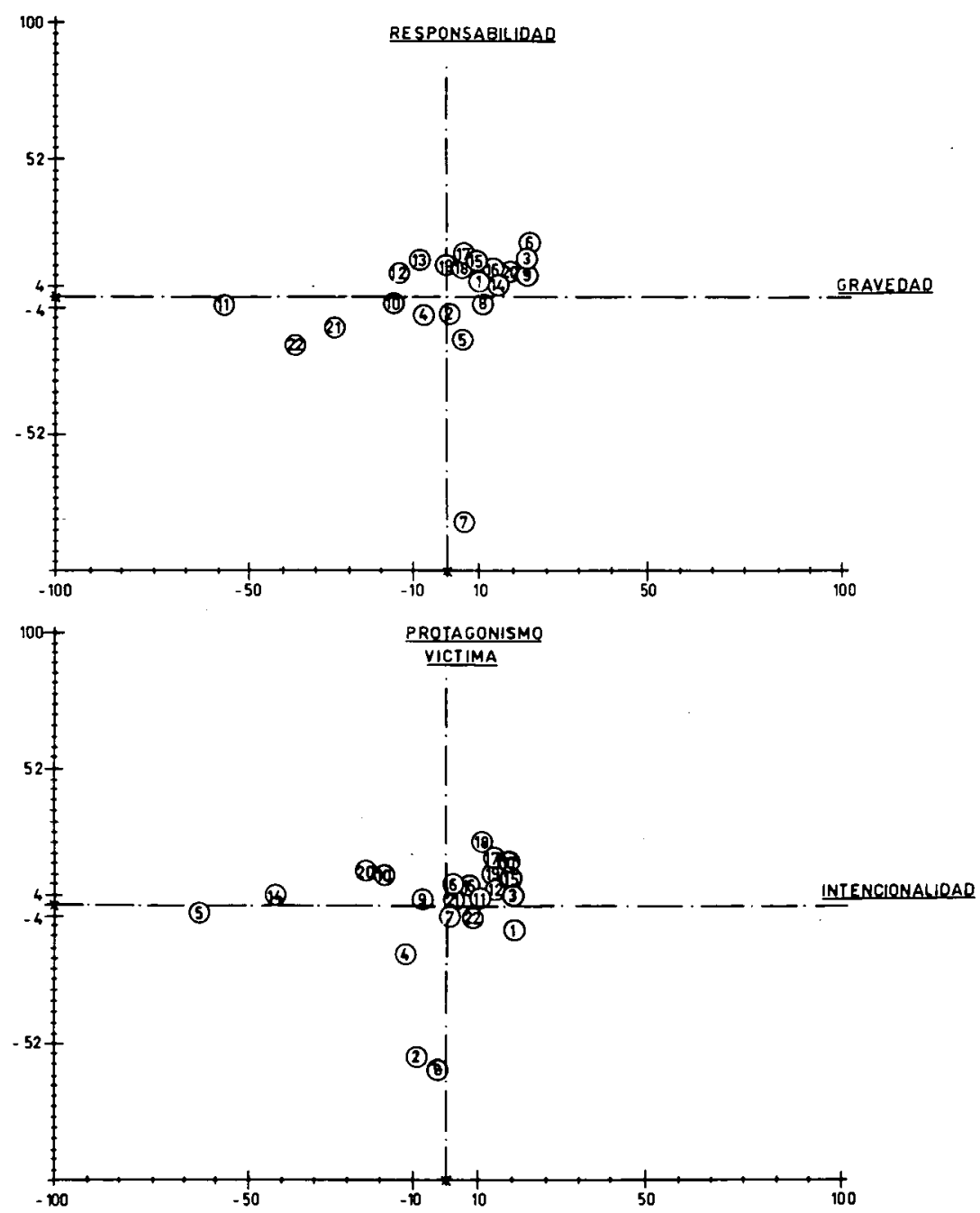
12. ESTAFA DEL TRACTOR
13. TIRON DE UN BOLSO
14. MUERTE DEL MOTORISTA
15. SECUESTRO
16. MALOS tRatos a la MUJER
17. ROBO Y DESTROZO DE UN COCHE
18. INTIMIOACION CON NAVAJA
19. ROBO DE COCHES
20. BORRACHO OUE APUÑala
21. CHICOS OUE ROMPEN MACETAS
22. ROBO EN UN ALMACEN

Representación gráfica de los 22 episodios delictivos en el espacio perceptivo de las personas no vinculadas al sistema penal. 
que destroza un coche después de robar (17) o el hombre que da un tirón (13). La etiqueta que hemos elegido para esta dimensión es responsabilidad, puesto que viene definida por escalas como «el agresor es responsable/el agresor no es responsable» $(0,87)$, "el agresor es culpable/el agresor no es culpable" $(0,87)$, «en provecho propio/en provecho ajeno» $(0,68)$ $\mathrm{y}$ "no me cae bien el agresor/me cae bien" $(0,53)$. El porcentaje de varianza explicado fue de un $14,6 \%$.

La tercera dimensión separa, por un lado, episodios como el del hijo que mata a su madre sin querer (5), la imprudencia punible del accidente (14), o del borracho que apunala a otra persona (20) y, por el otro, el tráfico de drogas (1), la trata de blancas (3), el secuestro (15) o el tirón (13). Esta dimensión la hemos denominado intencionalidad, explica un 13,1\% de la varianza, y está definida por las escalas «intencional/no intencional» $(0,79)$, «lo volverá a hacer/no lo volverá a hacer» $(0,72)$, «calculado/pasional» $(0,70)$, «no provocado/provocado» $(0,62)$ y, en menor medida, por «sin influencia del alcohol/con influencia» $(0,48)$, «es probable que pase/es raro que pase» $(0,43)$ y «sólo para hacer daño/para conseguir algo» $(0,21)$.

La cuarta dimensión distingue entre el episodio del coreano que apuñala a quien le había robado (8) o el del chico que golpea y roba a unos ladrones (2), frente al robo con intimidación (18), el tirón (13), o el chico que destroza un coche después de robar en su interior (17). Esta dimensión explica un $7,3 \%$ de la varianza y recibe el nombre de protagonismo de la víctima, ya que está definida por las escalas «empezó el agresor/empezó la víctima» $(0,87)$ y «me cae bien la víctima/no me cae bien la víctima» $(0,55)$.

Las correlaciones entre las dimensiones fueron muy pequeñas, salvo en el caso de la gravedad y la responsabilidad $(0,22)$, la intencionalidad y la responsabilidad $(0,25)$ y la responsabilidad y el protagonismo de la víctima $(0,25)$.

A continuación presentamos la tabla VI resumen de los nombres que hemos dado a cada una de las dimensiones obtenidas en cada población.

\section{Percepción de diferentes tipos de delitos}

Con el propósito de comprobar si los sujetos diferenciaban entre tres tipos de delitos - contra la propiedad, contra las personas y contra la honestidad - tradicionales en el Derecho Penal, se llevó a cabo un análisis discriminante en cada población.

Las variables discriminantes venían dadas por las puntuaciones de cada episodio en cada una de las dimensiones obtenidas a través del INDSCAL. La variable clasificatoria tipo de delito se estableció siguiendo el Código Pe-

\section{TABLA VI}

Lista de las etiquetas de las dimensiones obtenidas en el INDSCAL en cada población

\begin{tabular}{|c|c|c|c|}
\hline Dimensiones & Delincuentes & Profesionales & Control \\
\hline $\begin{array}{l}1 \\
2 \\
3 \\
4\end{array}$ & $\begin{array}{l}\text { Gravedad } \\
\text { Responsabilidad } \\
\text { Intencionalidad } \\
\text { Atenuantes }\end{array}$ & $\begin{array}{l}\text { Gravedad } \\
\text { Intencionalidad } \\
\text { Responsabilidad } \\
\text { Protagonismo } \\
\text { de la víctima }\end{array}$ & $\begin{array}{l}\text { Gravedad } \\
\text { Responsabilidad } \\
\text { Intencionalidad } \\
\text { Protagonismo } \\
\text { de la víctima }\end{array}$ \\
\hline
\end{tabular}


nal vigente, asignando a cada episodio uno de los tres valores a partir de las características del mismo. Son delitos contra la propiedad los robos, estafas, hurtos, etc. Los delitos contra las personas incluyen, por ejemplo, el homicidio y las lesiones, mientras que en la categoría de delitos contra la honestidad cabría citar la violación, los abusos deshonestos o el estupro. En aquellos casos en los que el episodio se refería al mismo tiempo a dos o tres tipos de delitos se primó el más saliente. La clasificación quedó tal como aparece en la tabla VII.

TABLA VII

Lista de los episodios asignados a cada tipo de delito

\begin{tabular}{rc}
\hline Tipo de delito & Episodios \\
\hline Delitos contra la propiedad & $11,12,13,17,18,19,21,22$ \\
Delitos contra las personas & $1,2,5,7,8,9,10,14,15,20$ \\
Delitos contra la honestidad & $3,4,6,16$ \\
\hline
\end{tabular}

\section{Delincuentes}

En el caso de los delincuentes se aislaron dos funciones discriminantes, de las cuales sólo una fue significativa $\left(\chi^{2}(8)=18,67, p<0,01\right)$. Esta función, que explica un $90,28 \%$ de la varianza, correlaciona con la dimensión de gravedad $(-0,69)$ e intencionalidad $(0,37)$ y permite diferenciar entre los delitos contra la propiedad $(1,50)$, por un lado, y los delitos contra las personas $(-0,93)$ y contra la honestidad $(-0,66)$ por el otro. Los delitos contra las personas y la honestidad son vistos como más graves en sí mismos, pero con menos intencionalidad que los delitos contra la propiedad.

\section{Profesionales}

Los profesionales también distinguen entre los tres tipos de delitos. De las dos funciones discriminantes sólo una fue significativa $\left(\chi^{2}(8)=20,35\right.$, $p<0,00$ ). Esta función explica por sí sola un $95,41 \%$ de la varianza, correlaciona principalmente con las dimensiones de gravedad $(-0,57)$ e intencionalidad $(0,46)$ y distingue entre los delitos contra las personas $(-1,21)$ y contra la honestidad $(-0,25)$ frente a los delitos contra la propiedad $(1,64)$. Los delitos contra la propiedad descritos son vistos como menos graves pero más intencionales que los delitos contra la honestidad y las personas.

\section{Grupo no vinculado al sistema penal}

En el grupo de personas no relacionadas con el sistema penal también fue una la función discriminante significativa, de las dos aisladas $\left(\chi^{2}(8)=21,45, p<0,00\right)$. Esta función explica por sí sola un $94,58 \%$ de la varianza y correlaciona con la gravedad $(-0,52)$, la intencionalidad $(0,42)$ y con el protagonismo de la víctima $(0,31)$. Esta función discrimina entre los delitos contra la propiedad $(1,71)$, por un lado, y los delitos contra las personas $(-1,20)$ y la honestidad $(-0,41)$, por el otro. En los delitos con- 
tra las personas y la honestidad son, una vez más, los más graves y los menos intencionales. En este caso se percibe además un mayor protagonismo de la víctima, frente a los delitos contra la propiedad.

\section{DISCUSION}

En líneas generales el espacio perceptivo de los delincuentes, los profesionales y las personas no vinculadas al sistema penal, es semejante. Los sujetos de nuestro estudio consideran como dimensiones más salientes la gravedad, la responsabilidad del agresor y la intencionalidad del hecho. La diferencia básica está quizá en que los delincuentes tienen en cuenta una dimensión de atenuantes, mientras los profesionales y los sujetos no vinculados al sistema penal se centran más en el protagonismo de la víctima.

Estos resultados se oponen parcialmente a nuestras expectativas, dejando claro que existe una serie de dimensiones de juicio que son compartidas por todos estos sujetos y que trascienden sus diferencias subculturales. Los datos de que disponemos no son suficientes, sin embargo, para afirmar que tales dimensiones son compartidas por la comunidad social de la que hemos extraído estas muestras. Tampoco podemos hablar de la percepción del delito en el sistema penal hasta que dispongamos de datos procedentes de otros grupos implicados en la administración de la justicia (por ejemplo abogados, fiscales).

En cuanto a los estudios previos, sólo la dimensión de intencionalidad coincide con las halladas por Forgas (1980; Forgas y cols., 1980), aun cuando muchas de las escalas utilizadas por nosotros son semejantes a las de este autor. En el caso del trabajo realizado por Sherman y Dowle (1974), tampoco se pudo establecer paralelismo alguno. Este hecho puede explicarse en función de la permeabilidad de los espacios perceptivos a la subcultura (por ejemplo Forgas, 1976), la cultura (por ejemplo Forgas y Bond, 1985) y el grupo (por ejemplo Forgas, 1981b) al que pertenecen los sujetos. Habiamos apuntado ya que todas las investigaciones de las que tenemos noticias se han llevado a cabo en el mundo anglosajón.

El haber aislado una dimensión de gravedad contradice parcialmente los estudios anteriores en los que se propugnaba que este concepto no era unidimensional sino multidimensional. Sin embargo, las escalas de gravedad y cantidad de castigo, que son las más que correlacionan con esta dimensión, aun cuando se sitúan muy cerca una de la otra en el espacio perceptivo, no coinciden, tal como habían puesto de manifiesto los datos obtenidos en otras investigaciones (por ejemplo, Martín, 1984). También las escalas de responsabilidad y la culpa se sitúan muy próximas, pero en ningún caso son intercambiables. La intencionalidad, por su parte, constituye otra dimensión independiente, pero correlaciona con la responsabilidad en los tres grupos. Este resultado coincide con investigaciones sobre juicios morales, en las que se concluye que la intencionalidad es el factor que explica la mayor parte de la varianza en el juicio, aisladamente o en interacción con otras variables (Martín, 1984).

Respecto a la utilización de los atenuantes por parte de los delincuentes, y del protagonismo de la víctima por pate de los profesionales y el grupo de personas no vinculadas al sistema penal, existen dos líneas de investigación que apoyan nuestros resultados. 
En primer lugar, tanto en el campo de la atribución, el juicio moral o en las investigaciones realizadas con jurados, se ha comprobado que la similitud percibida del observador con la víctima y/o agresor influye en su juicio (ver Martín, 1984, 1987b). Desde esta perspectiva, los delincuentes tenderán a verse más como agresores, necesitados de justificación o excusas, mientras que los otros dos grupos adoptarán más el papel de víctimas o de observadores. Nuestros datos son, pues, coherentes con estos planteamientos, y amplían los resultados obtenidos de forma puntual a una visión más global de la percepción social, indicando que hay indicios para creer que la hipótesis de la atribución defensiva (Shaver, 1970) actúa en la percepción de episodios sociales, de forma análoga a otros sesgos motivacionales (ver Forgas, 1982).

La segunda línea de investigación que apoya el escaso interés de los delincuentes por la víctima procede del ámbito criminológico. Diversos autores (ver, por ejemplo, Platt y Prout, 1987) han puesto de manifiesto las dificultades de los delincuentes, frente a los no delincuentes, para adoptar las perspectivas de otras personas. A partir de estas formulaciones se han desarrollado programas de prevención y reeducación, altamente efectivos (ver, por ejemplo, Ross y Fabiano, 1985), en los que se enfatiza la falta de capacidad empática del delincuente respecto a la víctima, dentro de un cuadro carencial más general que se circunscribe a los procesos de percepción $y$ razonamiento en interacciones sociales cotidianas.

En cuanto a la contribución específica de cada dimensión a la varianza explicada, los profesionales y el grupo de personas no vinculadas al sistema penal coinciden respecto a la dimensión de gravedad, protagonismo de la víctima y responsabilidad, aunque difieren respecto a la intencionalidad, más importante para los profesionales. Los delincuentes, por su parte, siguen la pauta del grupo de personas no vinculadas al sistema penal, aunque conceden mucho más importancia a la gravedad y menos importancia a la responsabilidad e intencionalidad. La última dimensión de atenuantes se equipara a la de protagonismo de las víctimas en los otros dos grupos.

No es de extrañar que los profesionales den mayor peso a la intencionalidad, ya que es un requisito contenido en el párrafo primero artículo 1 del Código Penal: «Son delitos o faltas las acciones y omisiones dolosas o culposas penadas por la ley.»Y por si no quedara claro con esto, en el párrafo segundo de dicho artículo se especifica: «No hay pena sin dolo o culpa.» Si no hay intencionalidad no hay delito y, si no lo hay, no tiene sentido determinar hasta qué punto el autor del hecho en cuestión es responsable o no. Para los legos en Derecho, sin embargo, el proceso no es el mismo. En los estudios realizados en el campo de la atribución de responsabilidad por conductas que causan daño (ver Hernández y Martín, 1988) se ha puesto de manifiesto que el proceso atribucional no sigue un modelo secuencial lógico de este tipo y que, en determinadas circunstancias, las personas pueden incluso atribuir más responsabilidad por un acto accidental que por uno intencional (por ejemplo Walster, 1966). En la base de esta «falta de lógica" están los sesgos motivacionales a los que ya hemos hecho referencia anteriormente.

El énfasis puesto por los delincuentes en la dimensión de gravedad puede explicarse desde los estudios sobre desarrollo moral (ver Martín, 1984). Las personas en «estadios más primitivos» del desarrollo se centran más en 
la gravedad de las consecuencias y del hecho en sí mismo, que en la intencionalidad del mismo. Sin embargo, nosotros no explicamos esta diferencia de los delincuentes - frente a los no delincuentes - apelando, tal como hace Kohlberg y sus seguidores, a una inferioridad moral. Creemos que se trata más bien de diferencias en las destrezas cognitivas requeridas en la percepción y evaluación de situaciones interpersonales, en la línea de los trabajos criminológicos comentados más arriba, y que pueden llevar a la configuración de ideologías éticas particulares (ver Martín y Rodríguez, 1986). La adecuación o inadecuación de tales diferencias depende más de criterios pragmáticos de funcionamiento social efectivo del sujeto, que de la comparación con estándares de verdad absoluta.

Siguiendo con nuestro estudio, una representación gráfica de las puntuaciones de cada una de las escalas bipolares en el espacio perceptivo, semejante a la que hemos presentado para los episodios, permitiría extraerse conclusiones muy interesantes respecto a la semántica de los términos utilizados y, consiguientemente, sobre la intercambiabilidad de las variables dependientes usadas tradicionalmente. Asimismo, es posible establecer multitud de categorías a partir de las cuales las personas encuestadas han agrupado los episodios, así como las características que comparten cada una de dichas categorías. Sin embargo, nos limitaremos a considerar sólo tres categorías de las establecidas en el Código Penal español: delitos contra la propiedad, contra las personas y contra la honestidad.

Tanto los profesionales como los delincuentes y el grupo de personas no vinculadas al sistema penal, distinguen los delitos contra la propiedad de los delitos contra las personas y la honestidad, a la hora de evaluarlos. Los análisis discriminantes que comentamos anteriormente, dejan patente cómo todos los sujetos coinciden al considerar que los delitos contra la propiedad son los menos graves pero los más intencionales. En el grupo de personas no vinculadas con el sistema penal se percibe además un menor protagonismo de la víctima en este tipo de delitos. Dado el reducido número de episodios delictivos que fueron etiquetados como delitos contra la honestidad, no es extraño que la función que contrapone este grupo a los dos restantes, no sea significativa. Sin embargo, tanto los centroides dados por las funciones no significativas como las representaciones gráficas de los espacios perceptivos (ver figs. 1, 2 y 3) muestran que los sujetos de los tres grupos tienden a agrupar y a percibir diferencialmente los delitos contra la honestidad, sobre todo en lo que se refiere a los episodios 3,6 y 16 . El episodio 4, que trata sobre una "presunta" violación, no se percibe como formando parte de la misma categoría, quizás porque la mayoría de los sujetos se inclinaron a pensar que era una falsa acusación y no una violación real.

Hasta aquí nos hemos centrado en la discusión de los principales resultados de este estudio. Para terminar, y aunque la determinación del origen consensual o político de la ley no sea uno de los objetivos de esta investigación, nos gustaría apuntar algo al respecto.

En sociedades como la actual, caracterizadas por su diversidad, nos parece utópico pretender que la ley, como otros productos sociales, pueda reflejar los valores y costumbres de todos. De "una mayoría», tal vez, pero no de todos. Parece más plausible pensar que los legisladores, aunque se les suponga toda la buena voluntad del mundo, reflejan la visión de aquéllos más próximos a ellos. Algunas veces esa visión es compartida por otros 
grupos sociales, y otras veces no. Pero una vez instituida, la ley será acatada por el ciudadano de a pie, por razones que no hace falta explicitar. En Psicología Social, se sabe desde hace mucho tiempo que la ejecución repetida de una conducta que en principio se opone a las creencias del individuo le lleva, en determinadas circunstancias por supuesto, a cambiar sus ideas al respecto.

Los datos que hemos presentado en este estudio no son suficientes para aceptar o rechazar esta perspectiva - nunca pretendieron serlo-, pero proporcionan algunas claves para reflexionar sobre el tema. Las diferencias encontradas entre los grupos, especialmente entre los profesionales y los no profesionales, los delincuentes y los no delincuentes, no parecen apoyar el proceso de abajo a arriba. Es tarea de futuras investigaciones determinar el alcance de tales diferencias.

\section{Referencias}

Bblumer, R. (1969). Symbolic interactionism. Nueva York: Prentice-Hall.

CAMACHO, J., y HERNANDEZ, B. (1985). «Introducción al escalamiento multidimensional y sus aplicaciones en Psicología Ambiental», en Jiménez Burillo, F., y Aragonés J. I. (eds.): Introducción a la Psicología Ambiental (págs. 367-386). Madrid: Alianza.

Carroll, J. D., y Chang, J. J. (1970). "Analysis of individual differences in multidimensional scaling via an $\mathrm{N}$-way generalization of Eckart-Young decomposition". Psychometrika, 35: 283-319.

Cronbach, C. J., y Gleser, G. C. (1952). "Assessing similarity between profiles». Psychological Bulletin, 50: 456-473.

FLANAGAN, J. C. (1954). "The critical incident technique». Psychological Bulletin, 51: 327-358.

FORGAS, J. P. (1976). "The perception of social episodes: Categorial and dimensional representations in two social milieus". Journal of Personality and Social Psychology, 34: 199-209.

ForGAS, J. P. (1978). "Social episodes and social structure in an academic setting: The social environment of an intact group". Journal of Experimental Social Psychology, 14: 434-448.

FORGAS, J. P. (1979a). Social episodes: The study of interaction routines. Nueva York: Academic Press.

FORGAS, J. P. (1979b). «Multidimensional Scaling: A discovery method in social psychology", en Ginsburg, G. P (ed.): Emerging strategies in Social Psychology, (págs. 253-288). Nueva York: Wiley.

FORGAS, J. P. (1980a). «Images of crime: The perception of criminal incidents». International Journal of Psychology, 15: 287-299.

FORGAS, J. P. (1981a). Social cognition: Perspectives on everyday understanding. Nueva York: Academic Press.

FORGAS, J. P. (1981b). "Social episodes and group milieu: a study in social cognition". The British Journal of Social Psychology, 20: 77-88.

FORGAS, J. P. (1982). "Episode cognition: Internal representations of interaction routines", en Berkowitz, L. (ed.): Advances in Experimental Social Psybology (Vol. 15, págs. 59-101). Nueva York: Academic Press.

FORGAS, J. P., y BOND, M. H. (1985). "Cultural influences on the perception of interaction episodes". Personality and Social Psychology Bulletin, 11: 75-88.

FORGAS, J. P.; BROWN, L. B., y MENYHART, J. (1980). «Dimensions of aggression: The perception of aggressive episodes». British Journal of Social Psychology, 19: 215-227.

GARZÓN, A., y SEOANE, J. (1988). "Dimensiones politicas en Psicología Judicial», en Seoane, J. (ed.), Psicología Politica (págs. 306-330). Madrid: Pirámide.

HERNÁNDEZ, B., y MARTín, A. (1988). “La atribución de responsabilidad en la percepción del daño", en Barriga, S.; Blanco, A.; Ibánez, T.; Jiménez, F.; Morales, J. F.; Muné, F.; Rodríguez, A.; Seoane, A. J.; Serrano, G., y Torregrosa, J. R. (eds.): Procedente del Primer Congreso Nacional en Psicologia Social (págs. 381-384). Granada: Servicio de Publicaciones de la Universidad de Granada.

KruSKAL, J. B., y WISH, M. (1978). Multidimensional scaling. Nueva York: Sage.

MARTín, A. M. (1984). Juicios morales sobre conducta agresiva. Memoria de licenciatura no publicada, Universidad de la Laguna.

MARTín, A. M. (1987a). «La percepción social del delito. En Sancha, V.; Clemente, M., y Miguel, J. J. (eds.): Delincuencia: Teoria e investigación (págs. 355-368). Madrid: Alpe. 